\title{
On the Spectral Gap for Laplacians on Metric Graphs
}

\author{
P. KURASOV* \\ Dept. of Mathematics, Stockholm Univ., 106 91, Stockholm, Sweden
}

\begin{abstract}
We discuss lower and upper estimates for the spectral gap of the Laplace operator on a finite compact connected metric graph. It is shown that the best lower estimate is given by the spectral gap for the interval with the same total length as the original graph. An explicit upper estimate is given by generalizing Cheeger's approach developed originally for Riemannian manifolds.
\end{abstract}

DOI: $10.12693 /$ APhysPolA.124.1060

PACS: 03.65.Nk, 73.63.-b, 85.35.-p

\section{Introduction}

This note will describe some of recent developments in the spectral theory of quantum graphs. More precisely, we are going to discuss the spectral gap - the distance between two lowest eigenvalues - for the Laplace operator on metric graphs in connection to geometric and topological properties of the underlying graphs. Our approach can be generalized further to include Schrödinger operators with non-trivial potentials on the edges and many observed properties will be preserved. On the other hand, studying the case of zero potential (Laplacian) enables us to focus on spectral features determined by geometric and topological properties only. The spectral gap is not only important for stability properties for evolution equation on graphs, but prove to be an important measure of their connectivity [1]. Our goal is not only to describe recent results in the area, but to indicate few interesting directions of further research.

Quantum graphs - differential operators on metric graphs - is an important area of modern mathematical physics going through a rapid development during recent years. There are already three monographs devoted to the subject and we refer to these books for further references and historical remarks [2-4]. We just mention that the spectral gap for quantum graphs was discussed in $[5-9]$.

\section{Basic definitions}

Consider an arbitrary finite compact metric graph viewed as a collection of a finite set of compact intervals $E_{n}=\left[x_{2 n-1}, x_{2 n}\right], n=1,2, \ldots, N$ joined together at the vertices $V_{m}, m=1,2, \ldots, M$ identified with equivalent classes of end points $x_{j}, j=1,2, \ldots, 2 N$. In the corresponding Hilbert space

$$
L_{2}(\Gamma)=\oplus \sum_{n=1}^{N} L_{2}\left(E_{n}\right)
$$

consider the Laplace operator $L=-\frac{\mathrm{d}^{2}}{\mathrm{~d} x^{2}}$ defined on the functions $u$ from the Sobolev space $W_{2}^{2}\left(\Gamma \backslash \cup_{m=1}^{M} V_{m}\right)$ satisfying standard matching conditions at every vertex $V_{m}$ :

\footnotetext{
*e-mail: kurasov@math.su.se
}

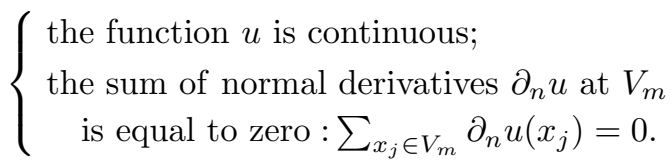

This differential operator to be denoted by $L(\Gamma)$ is called the standard Laplacian and is uniquely determined by the underlying graph $\Gamma$. The operator is self-adjoint, non-negative and the spectrum is pure discrete, since $\Gamma$ is formed by a finite number of compact intervals. The lowest eigenvalue $\lambda_{0}(\Gamma)$ is equal to zero and is non-degenerate, provided the graph is connected. The corresponding eigenfunction is just the constant function. The difference between the lowest two eigenvalues $\Delta(\Gamma) \equiv \lambda_{1}(\Gamma)-\lambda_{0}(\Gamma) \equiv \lambda_{1}(\Gamma)$ is called the spectral gap and will be the main object of our interest.

\section{Rayleigh estimate}

The Rayleigh quotient gives us an exact formula for the first eigenvalue

$$
\lambda_{1}(\Gamma)=\min _{u: \int_{\Gamma}} u(x) \mathrm{d} x=0 \quad \frac{\int_{\Gamma}\left|u^{\prime}(x)\right|^{2} \mathrm{~d} x}{\int_{\Gamma}|u(x)|^{2} \mathrm{~d} x},
$$

where the minimum is taken over all functions $u$ from the domain of the quadratic form which are orthogonal to the ground state eigenfunction $\psi_{0}(x) \equiv 1$. The domain of the quadratic form consists of all functions from the Sobolev space $W_{2}^{1}\left(\Gamma \backslash \cup_{m=1}^{M} V_{m}\right)$ which are continuous not only on the edges but at the vertices as well. The minimum is realized if we substitute instead of $u$ any eigenfunction corresponding to the eigenvalue $\lambda_{1}$. Taking an arbitrary function $w(x)$ (not necessarily orthogonal to the ground state) we get the following estimate for the first eigenvalue and therefore for the spectral gap:

$$
\Delta(\Gamma)=\lambda_{1}(\Gamma) \leq \frac{\int_{\Gamma}\left|w^{\prime}(x)\right|^{2} \mathrm{~d} x}{\int_{\Gamma}|w(x)|^{2} \mathrm{~d} x-\frac{1}{\mathcal{L}(\Gamma)}\left[\int_{\Gamma} w(x) \mathrm{d} x\right]^{2}} .
$$

The function $w$ appearing in the last formula should of course belong to the domain of the quadratic form of the Laplacian.

It follows that chopping a certain vertex into two or more (independent) vertices cannot lead to an increase of the spectral gap, but in many cases will lead to its decrease. Let us denote by $\Gamma^{\prime}$ the new graph appearing as a result of chopping: it has precisely the same edges 
as $\Gamma$, but to get back the vertices in $\Gamma$ one might need to join together several vertices in $\Gamma^{\prime}$.

The simplest case occurs when chopping of a vertex makes the graph not connected. Then the new graph consists of at least two connected components and the ground state becomes degenerate. Hence the spectral gap is zero.

If chopping does not make the graph disconnected, then the set of functions used in the Rayleigh quotient is increasing: it is not required anymore that the functions have the same values at the chopped vertices in $\Gamma^{\prime}$ corresponding to the same vertex in $\Gamma$. Since the set of allowed functions is increasing, the minimum may decrease or stay the same. If the minimizing function for $\Gamma^{\prime}$ has the same values in the chopped vertices, then requirement that the values are the same does not change the minimum.

This idea can be applied to get an explicit estimate for the spectral gap for Eulerian graphs - graphs with even valencies (degrees) of all vertices. The Euler theorem from $1736[10,11]$ states that for such a graph there exists a path $\mathcal{P}$ going along each edge precisely once ( $E u$ lerian path). The original graph $\Gamma$ can be transformed into the path $\mathcal{P}$ by chopping its vertices sufficiently many times. It follows that:

$$
\lambda_{1}(\mathcal{P}) \leq \lambda_{1}(\Gamma) .
$$

The path $\mathcal{P}$ can be identified with the loop-graph of length $\mathcal{L}(\Gamma)$ and its eigenvalues can be explicitly calculated

$$
\lambda_{j}=\left(\frac{2 \pi}{\mathcal{L}} j\right)^{2}, \quad j=1,2, \ldots
$$

The spectral gap $\Delta(\mathcal{P})=\lambda_{1}(\mathcal{P})$ is equal to $\left(\frac{2 \pi}{\mathcal{L}}\right)^{2}$ and provides a lower estimate for the spectral gap of $L(\Gamma)$. We have proven the following proposition.

Proposition 1. [Theorem 2 from [9]] Let $\Gamma$ be a connected finite compact metric graph with even valencies of all vertices and with the total length $\mathcal{L}(\Gamma)$ and $L(\Gamma)-$ the corresponding Laplace operator on $\Gamma$ defined on the functions from the Sobolev space $W_{2}^{2}\left(\Gamma \backslash\left\{V_{j}\right\}_{j=1}^{M}\right)$ satisfying standard matching conditions (2.1) at the vertices. Then the spectral gap for $L(\Gamma)$ can be estimated from below as follows:

$$
\Delta(\Gamma)=\lambda_{1}(\Gamma) \geq\left(\frac{2 \pi}{\mathcal{L}}\right)^{2} .
$$

This approach can be generalized to give a lower estimate for arbitrary (not necessarily Eulerian) graphs. In order to use the idea with the Eulerian path we may double all the edges getting a new graph $\Gamma^{*}$ with double total length $2 \mathcal{L}(\Gamma)$ and doubled valencies of all vertices. Any function on $\Gamma$ can be extended to $\Gamma^{*}$ by assigning the same values on every doubled edge as on the original one. In this way we get an upper estimate for $\lambda_{1}\left(\Gamma^{*}\right)$, which is at the same time a lower estimate for $\lambda_{1}(\Gamma)$ :

$$
\lambda_{1}\left(\Gamma^{*}\right) \leq \lambda_{1}(\Gamma) .
$$

Applying Proposition 1 to graph $\Gamma^{*}$ we get a new estimate for the spectral gap of $L(\Gamma)$ :
Proposition 2. [Theorem 1 from [7] and Theorem 1 from [9]] Let $\Gamma$ be a connected finite compact metric graph with the total length $\mathcal{L}(\Gamma)$ and $L(\Gamma)$ - the corresponding Laplace operator on $\Gamma$ defined on the functions from the Sobolev space $W_{2}^{2}\left(\Gamma \backslash\left\{V_{j}\right\}_{j=1}^{M}\right)$ satisfying standard matching conditions (2.1) at the vertices. Then the spectral gap for $L(\Gamma)$ can be estimated from below as follows:

$$
\Delta(\Gamma)=\lambda_{1}(\Gamma) \geq\left(\frac{\pi}{\mathcal{L}}\right)^{2} .
$$

The latter Proposition gives a lower estimate which is 4 times worth than the one in Proposition 1, but it is not required that the valencies of all vertices are even, i.e. that the graph is Eulerian. It follows that among all graphs with the same total length the graph formed by one edge has the lowest spectral gap. Similarly, Proposition 1 implies that among all Eulerian graphs with the same total length the spectral gap is minimal for the loop. It is clear that obtained estimates cannot be improved since they are sharp for the edge and loop graphs, respectively. On the other hand, to make graph Eulerian it is not always necessary to double all the edges - for certain classes of (non-Eulerian) graphs it might be enough to double just few edges leading to improved estimates in comparison with (3.6).

It is interesting to mention that Proposition 2 has also been proven (earlier) using symmetrization technique. The main idea of this approach is to use the first eigenfunction $\psi_{1}(\Gamma)$ to construct an admissible function on the interval of the same total length and use it in the Rayleigh estimate. That approach does not use geometric and topological properties of the underlying graph and can hardly be generalized to obtain the estimate from Proposition 1. On the other hand, it might be fruitful to combine the two methods to get better estimates for other special classes of graphs.

\section{Cheeger type estimate}

This section is devoted to the proof of an upper estimate for the spectral gap. We are going to use the Rayleigh quotient again, but the corresponding construction is more involved. Our goal is to study whether Cheeger's approach originally developed for the first nontrivial eigenvalue of the Laplace-Beltrami operator on a compact Riemannian manifold $M$ [12] can be generalized for quantum graphs. Cheeger's original estimate is obtained by considering all possible submanifolds $S$ dividing $M$ into two submanifolds with boundary $M_{1}$ and $M_{2}$ and the corresponding constant

$$
h=\inf _{\left(M_{1}, M_{2}, S\right)} \frac{\operatorname{Area}(S)}{\min \left\{\operatorname{Vol} M_{1}, \operatorname{Vol} M_{2}\right\}},
$$

where $\operatorname{Area}(S)$ and $\operatorname{Vol}\left(M_{j}\right)$ denote the corresponding volumes of manifolds of different dimensions. Then the first nontrivial eigenvalue satisfies

$$
\lambda_{1} \geq \frac{1}{4} h^{2} .
$$

One may prove a similar inequality for Laplacians on graphs, but the form of the inequality is different. Consider an arbitrary connected metric graph $\Gamma$ and its de- 
composition into three sets:

- two nonintersecting subgraphs $\Gamma_{1}$ and $\Gamma_{2}$;

- the set $S=\Gamma \backslash\left(\Gamma_{1} \cap \Gamma_{2}\right)$.

We assume that the set $S$ consists of a collection of edges with the following property: precisely one of the end points belongs to each of the subgraphs $\Gamma_{1}$ and $\Gamma_{2}$. One may view this triple $\left(S, \Gamma_{1}, \Gamma_{2}\right)$ as a cut of the original graph $\Gamma$ : one cuts the graph along the edges belonging to $S$ so that the original graph falls into at least two parts $\Gamma_{1}$ and $\Gamma_{2}$. Let us note that we do not assume that $\Gamma_{1}$ and $\Gamma_{2}$ are connected. Such a cut of a metric graph will be called proper. Observe that proper cuts do not allow to cut the graph along the loops.

With any cut $\left(S, \Gamma_{1}, \Gamma_{2}\right)$ as described above let us associate the quotient (Cheeger's quotient)

$$
c_{\left(S, \Gamma_{1}, \Gamma_{2}\right)}(\Gamma)=\frac{\mathcal{L}(\Gamma) \sum_{E_{n} \in S} \ell_{n}^{-1}}{\mathcal{L}\left(\Gamma_{1}\right) \mathcal{L}\left(\Gamma_{2}\right)},
$$

where $\ell_{n}=x_{2 n}-x_{2 n-1}$ denotes the length of the interval $E_{n}$. Here $\mathcal{L}(\Gamma)$ is the total length (volume) of the original graph $\Gamma$ and $\mathcal{L}\left(\Gamma_{1,2}\right)$ are the total lengths (volumes) of the two components into which the graph $\Gamma$ is divided. The quantity $\sum_{E_{n} \in S} \ell_{n}^{-1}$ is a certain measure (volume) of the part of $\Gamma$ which is cut away.

Consider the function $w$ defined as follows:

$$
w(x)=\left\{\begin{array}{l}
1, \quad x \in \Gamma_{1}, \\
-1, \quad x \in \Gamma_{2}, \\
\ell_{n}^{-1}\left[-\operatorname{dist}\left(x, \Gamma_{1}\right)+\operatorname{dist}\left(x, \Gamma_{2}\right)\right], \quad x \in E_{n} \subset S,
\end{array}\right.
$$

where the distances $\operatorname{dist}\left(x, \Gamma_{j}\right), j=1,2$ are calculated along the corresponding interval $x \in E_{n}$. Here it is very important that every edge from $S$ builds a certain bridge between $\Gamma_{1}$ and $\Gamma_{2}$. The continuous function $w$ is constructed in such a way that it is equal to \pm 1 on $\Gamma_{1}$ and $\Gamma_{2}$ and is linear on the edges connecting $\Gamma_{1}$ and $\Gamma_{2}$. The mean value of the function might be different from zero. In that case the function $w$ has to be modified so that it will be orthogonal to the ground state. Consider then the function $u$ which is not only continuous, but also orthogonal to the ground state

$$
u(x)=w(x)-\mathcal{L}(\Gamma)^{-1}\langle w, 1\rangle_{L_{2}(\Gamma)} .
$$

The Rayleigh quotient for the function $f$ gives an upper estimate for the spectral gap.

Let us calculate the Dirichlet integral and the norm of $u$ :

$$
\begin{aligned}
& \left\|u^{\prime}\right\|_{L_{2}(\Gamma)}^{2}=\left\|w^{\prime}\right\|_{L_{2}(\Gamma)}^{2} \\
& \quad=\sum_{E_{n} \in S} \int_{E_{n}}\left(-2 \ell_{n}^{-1}\right)^{2} \mathrm{~d} x=4 \sum_{E_{n}} \ell_{n}^{-1}, \\
& \|u\|_{L_{2}(\Gamma)}^{2}=\|w\|_{L_{2}(\Gamma)}^{2}-\mathcal{L}(\Gamma)^{-1}\langle w, 1\rangle^{2} \geq \mathcal{L}\left(\Gamma_{1}\right) \\
& \quad+\mathcal{L}\left(\Gamma_{2}\right)-\mathcal{L}(\Gamma)^{-1}\left[\mathcal{L}\left(\Gamma_{1}\right)-\mathcal{L}\left(\Gamma_{2}\right)\right]^{2} \geq 4 \frac{\mathcal{L}\left(\Gamma_{1}\right) \mathcal{L}\left(\Gamma_{2}\right)}{\mathcal{L}(\Gamma)} .
\end{aligned}
$$

This gives the following upper estimate for $\lambda_{1}(\Gamma)$ :

$$
\lambda_{1}(\Gamma) \leq c_{\left(S, \Gamma_{1}, \Gamma_{2}\right)}(\Gamma),
$$

where we use (4.2).

Theorem 1. Let $\Gamma$ be a connected finite compact metric graph without loops, then the spectral gap $\Delta(\Gamma)$ for the corresponding standard Laplacian can be estimated from above by Cheeger's constant $C(\Gamma)$ :

$$
\begin{aligned}
& \Delta(\Gamma)=\lambda_{1}(\Gamma) \leq C(\Gamma) \\
& \quad \equiv \underset{\left(S, \Gamma_{1}, \Gamma_{2}\right)-\text { proper cut of } \Gamma}{\min } \frac{\mathcal{L}(\Gamma) \sum_{E_{n} \in S} \ell_{n}^{-1}}{\mathcal{L}\left(\Gamma_{1}\right) \mathcal{L}\left(\Gamma_{2}\right)} .
\end{aligned}
$$

Proof. The result follows immediately from estimate (4.5) taking into account that the proper cut $\left(S, \Gamma_{1}, \Gamma_{2}\right)$ dividing $\Gamma$ into disconnected components is arbitrary.

We have already mentioned that $\mathcal{L}(\Gamma)$ and $\mathcal{L}\left(\Gamma_{1,2}\right)$ can be considered as volumes of the graph $\Gamma$ and its subgraphs $\Gamma_{1,2}$. The minimum is realized if the subgraphs $\Gamma_{1}$ and $\Gamma_{2}$ have approximately the same volumes $\mathcal{L}\left(\Gamma_{1,2}\right) \sim \frac{1}{2} \mathcal{L}(\Gamma)$. The set $S$ should be chosen with the minimal possible measure $\sum_{E_{n} \in S} \ell_{n}^{-1}$, which means that to get the best estimate one should cut $\Gamma$ along few long edges. On the other hand, choosing long edges for $S$ decreases the denominator $\mathcal{L}\left(\Gamma_{1}\right) \mathcal{L}\left(\Gamma_{2}\right)$. The choice of the optimal proper cut is not obvious and is worth to study.

\section{Acknowledgments}

The author would like to thank the referee for an extremely careful reading of the manuscript.

\section{References}

[1] M. Fiedler, Czechoslov. Math. J. 23, 298 (1973).

[2] G. Berkolaiko, P. Kuchment, Introduction to Quantum Graphs, AMS, Providence 2013.

[3] P. Kurasov, "Quantum Graphs: Spectral Theory and Inverse Problems", in preparation.

[4] O. Post, Lecture Notes Math., Vol. 2039, Springer, Heidelberg 2012.

[5] G. Berkolaiko, P. Kuchment, Proc. Symp. Pure Math. 84, 117 (2012).

[6] P. Exner, M. Jex, Phys. Lett. A 376, 713 (2012).

[7] L. Friedlander, Ann. Inst. Fourier (Grenoble) 55, 199211 (2005).

[8] P. Kurasov, G. Malenova, S. Naboko, J. Phys. A, Math. Theor. 46, 275309 (2013).

[9] P. Kurasov, S. Naboko, accepted for publication in J. Spectral Theory.

[10] L. Euler, "Solutio problematis ad geometriam situs pertinentis", Comment. Academiae Sci. I. Petropolitanae 8, 128 (1736).

[11] C. Hierholzer, Chr. Wiener, "Über die Möglichkeit, einen Linienzug ohne Wiederholung und ohne Unterbrechung zu umfahren", Math. Ann. 6, 3032 (1873) (in German).

[12] J. Cheeger, in: Problems in Analysis, Ed. R.C. Gunning, Princeton University Press, New Jersey 1970, p. 195. 